\title{
Optimizing the Spectral Efficiency in mmWave Massive SU-MIMO Systems Using Hybrid Processing
}

\author{
Alvaro Javier Ortega
}

\begin{abstract}
In this letter, a methodology to optimize the spectral efficiency in downlink massive single-user multiple-input multiple-output (SU-MIMO) millimeter-wave (mmWave) systems is proposed. Making use of a hierarchical strategy, four optimization sub-problems are formulated, whose solutions and derivations are strongly related to each other. This fact produces efficient coordination between the parties involved such that higher spectral efficiency is achieved. Therefore, the main feature of the proposed methodology relies on the coordination of the proposed RF and passband beamformer of both the transmitter and the receiver. This fact produced that the proposed hybrid processing surpasses the considered hybrid processings and even the considered fully-digital technique in the simulated scenarios.
\end{abstract}

Index Terms-Millimeter-wave, hybrid precoder, hybrid combiner, spectral efficiency, SU-MIMO

\section{INTRODUCTION}

Millimeter-wave (mmWave) wireless communications have been demonstrated as a promising candidate to solve the spectrum congestion problem and to increase the data rate of the system. In mmWave scenarios, a massive number of antennas is required in order to compensate for the adverse channel conditions. However, fully digital signal processing techniques require a radio frequency $(\mathrm{RF})$ chain dedication per antenna, which is impractical in mmWave scenarios due to manufacturing cost and power consumption. Hybrid alternatives have been considered as a promising technology to provide a compromise between hardware complexity and system performance by reducing the number of RF chains. A large number of hybrid precoder designs have been proposed, e.g. [1] and its references. The present letter is focused on the spectral efficiency in mmWave scenarios with massive single-user MIMO (SU-MIMO). References [2]-[4] present a hierarchical strategy to divide the hybrid processing problem into analog and digital subproblems. In this strategy, the analog part of the hybrid precoder/combiner is typically computed first. Then, the digital part of the hybrid precoder/combiner is obtained by considering the analog part as fixed. To consider another order, an alternative optimization algorithm is necessary, e.g., [5]. However, this methodology considerably increases the complexity of the system. The challenge of this hierarchical strategy is to find a correct formulation for each

A. Ortega is with the Institute of Science and Technology Sidia, Manaus, Brazil, e-mail: alvaro.ortega@sidia.com. This work was partially supported by Samsung Eletrônica da Amazônia Ltda., under the auspice of the informatic law no $8.387 / 91$.

Digital Object Identifier: $10.14209 /$ jcis.2021.14 optimization subproblem so that joining their solutions produces better results. Reference [5] describes a very interesting method to optimize the achievable rate of the system. The authors consider a recursive method to alternatively compute the analog and digital beamformer. Although this methodology offers an attractive solution to optimize the spectral efficiency of the system, its computational complexity is questionable, especially when the analog beamformer is obtained at the cost of a high complex iterative algorithm, whose complexity increases as the number of antennas is large. For this reason, in the simulated scenarios considered by the authors, the number of antennas is limited to six antennas. This fact makes that this type of solution is prohibited for scenarios with massive MIMO. The present letter provides a more appropriate manner to further optimize the spectral efficiency in mmWave massive SU-MIMO systems. Instead of implementing an alternate optimization, the design of the hybrid combiner is approached together with the hybrid precoder aiming to maximize the spectral efficiency. The proposed methodology considers a hierarchical strategy, where four optimization subproblems are defined, whose analysis and derivation are detailed. Furthermore, for comparison purposes, an optimal fully-digital signal processing technique is used as an evaluation benchmark. The considered digital technique computes a fully-digital matched filter $(\mathrm{MF})$ as a precoder and a para-unitary matrix as a combiner. Since the MF can maximize the SNR, it reaches an optimum spectrum efficiency for single-user MIMO scenarios [6]. Even though, it is necessary to highlight that the considered fully-digital technique does not provide the maximum spectrum efficiency because there are other more sophisticated digital techniques that can reach better results. However, the rate performance of MF is very useful as a benchmark in SU-MIMO scenarios, because it is challenging to reach or even overcome by hybrid techniques. Furthermore, other references, e.g., [7]-[9], have used similar comparison approaches. Numerical results evidence the proposed methodology no only overcomes the considered hybrid techniques but also the considered fully-digital technique. Generally, there is a performance gap between the hybrid and fully digital techniques. However, the proposed methodology gets to eliminated this performance gap and obtain a slight gain in the simulated scenarios.

\section{SYSTEM MODEL}

In the transmitter or base station (BS), the hybrid precoder, $\mathbf{F}$, can be represented by the product between the RF beam- 
former, $\mathbf{F}_{R F}$, and the baseband beamformer, $\mathbf{F}_{B B}$. On the other side, the receiver or mobile station (MS) is equipped with $N_{r}$ antennas and $N_{R F_{r}}$ RF chains to process $N_{s}$ streams. The BS has $N_{t}$ antennas and sends $N_{s}$ streams simultaneously using $N_{R F_{t}}$ RF chains. Furthermore, power normalization is satisfied such that $\left\|\mathbf{F}_{R F} \mathbf{F}_{B B}\right\|_{F}^{2}=E_{T}$, where $E_{T}$ represents the total energy available at the BS for transmission.

The received signal by the user, $\mathbf{r}$, is expressed as

$$
\mathbf{r}=\mathbf{H F}_{R F} \mathbf{F}_{B B} \mathbf{s}+\mathbf{n}
$$

where $\mathbf{H}$ denotes the channel matrix from the BS to the user satisfying $\mathbb{E}\left[\|\mathbf{H}\|_{F}^{2}\right]=N_{t} N_{r}, \mathbf{n}$ is a complex Gaussian noise vector with zero-mean and covariance matrix $\sigma_{n}^{2} \mathbf{I}_{N_{r}}$; $\mathbf{s}$ is the data stream vector with $\mathbb{E}\left[\mathbf{s s}^{H}\right]=\mathbf{I}_{N_{s}}$ and whose entries belong to a constellation $\mathbb{Q}$. The analog part of the precoder, $\mathbf{F}_{R F}$, is implemented by phase shifters, satisfying $\left\|\left(\mathbf{F}_{R F}\right)_{i, j}\right\|=\frac{1}{\sqrt{N_{t}}}$. The processed received signal is

$$
\mathbf{y}=\mathbf{W}_{B B}^{H} \mathbf{W}_{R F}^{H} \mathbf{H F}_{R F} \mathbf{F}_{B B} \mathbf{s}+\mathbf{W}_{B B}^{H} \mathbf{W}_{R F}^{H} \mathbf{n}
$$

where $\mathbf{W}_{B B}$ and $\mathbf{W}_{R F}$ are the baseband and the RF combining matrix, respectively. Similarly to the RF beamformer in the $\mathrm{BS}, \mathbf{W}_{R F}$ is implemented using phase shifters and therefore $\left\|\left(\mathbf{W}_{R F}\right)_{i, j}\right\|=\frac{1}{\sqrt{N_{r}}}$. In addition, the signal-to-noise ratio $(\mathrm{SNR})$ is defined as $\mathrm{SNR}=\frac{E_{T}}{\sigma_{n}^{2}}$.

\section{CHANNEL MODEL}

We consider the Saleh-Valenzuaela mmWave channel model for indoor scenarios, which is given by

$$
\mathbf{H}=\sqrt{\frac{N_{t} N_{r}}{N_{p}}} \sum_{p=1}^{N_{p}} \alpha_{k, p} \mathbf{a}_{N_{r}}\left(\phi_{k, p}^{r}, \theta_{k, p}^{r}\right) \mathbf{a}_{N_{t}}\left(\phi_{k, p}^{t}, \theta_{k, p}^{t}\right)^{H}
$$

where $N_{p}$ is the number of multi-path components in the channel; $\alpha_{k, p} \backsim \mathcal{C N}(0,1)$ is the complex gain of the $p$-th multi-path component in the channel for the $k$-th user, whereas $\phi_{k, p}^{r}\left(\theta_{k, p}^{r}\right)$ and $\phi_{k, p}^{t}\left(\theta_{k, p}^{t}\right)$ are its azimuth (elevation) angles of arrival and departure, respectively [10]. We consider the use of an uniform planar array (UPA) formed by $N_{t}=N_{t_{h}} N_{t_{v}}$ $\left(N_{r}=N_{r_{h}} N_{r_{v}}\right)$ antennas, $N_{t_{h}}\left(N_{r_{h}}\right)$ antennas in the horizontal side and $N_{t_{v}}\left(N_{r_{v}}\right)$ antennas in the vertical side, with the antenna spacing of half wave length at the transmitter (receiver), whose response is given by (4) and (5).

$$
\begin{aligned}
\mathbf{a}_{N_{t}}(\phi, \theta) & =\mathbf{a}_{N_{t_{h}}}(\pi \cos (\phi) \sin (\theta)) \otimes \mathbf{a}_{N_{t_{v}}}(\pi \cos (\theta)) \\
\mathbf{a}_{M}(\psi) & =\frac{1}{\sqrt{M}}\left[1, e^{j \psi}, \ldots, e^{j(M-1) \psi}\right]^{T} \in \mathbb{C}^{M \times 1}
\end{aligned}
$$

\section{HYBRID PRECODER DESIGN}

The achievable rate, $R$, for the system described above is given by

$$
R=\log _{2}\left|\mathbf{I}_{N_{s}}+\mathbf{K}^{-1} \mathbf{W}^{H} \mathbf{H F F} \mathbf{F}^{H} \mathbf{H}^{H} \mathbf{W}\right|
$$

where $\mathbf{K}=\sigma_{n}^{2} \mathbf{W}^{H} \mathbf{W}$ and $\mathbf{W}=\mathbf{W}_{R F} \mathbf{W}_{B B}$. Thus, the following optimization problem is defined

$$
\begin{aligned}
& \mathbf{F}^{*}=\underset{\mathbf{F}}{\arg \max } R \\
& \text { s.t. }\left\|\left(\mathbf{F}_{R F}\right)_{i, j}\right\|=1 / \sqrt{N_{t}},\|\mathbf{F}\|_{F}^{2} \leq E_{T}
\end{aligned}
$$

As proved in [8] the optimization problem of maximizing the achievable rate can be simplified and formulated as

$$
\begin{aligned}
& \mathbf{F}^{*}=\underset{\mathbf{F}}{\arg \max } \operatorname{tr}\left\{\mathbf{F}^{H} \mathbf{H}^{H} \mathbf{W} \mathbf{W}^{H} \mathbf{H F}\right\} \\
& \text { s.t. }\left\|\left(\mathbf{F}_{R F}\right)_{i, j}\right\|=1 / \sqrt{N_{t}},\|\mathbf{F}\|_{F}^{2} \leq E_{T}
\end{aligned}
$$

Trying to solve directly the optimization problem described in (8) is mathematically challenging. To develop a feasible solution, a hierarchical strategy is adopted to divide the hybrid precoding problem into two sub-problems. Subsection IV-A and IV-B present the design of the RF and baseband beamformer, respectively.

\section{A. Analog part of the hybrid precoder}

Considering that only analog processing is available in the base station, the problem in (8) can be rewritten as

$$
\begin{aligned}
\mathbf{F}_{R F}^{*} & \underset{\mathbf{F}_{R F}}{\arg \max } \operatorname{tr}\left\{\mathbf{F}_{R F}^{H} \mathbf{H}^{H} \mathbf{W} \mathbf{W}^{H} \mathbf{H} \mathbf{F}_{R F}\right\} \\
\text { s.t. } & \left\|\left(\mathbf{F}_{R F}\right)_{i, j}\right\|=1 / \sqrt{N_{t}}
\end{aligned}
$$

To solve the above problem, we propose two solutions. The first solution, named hereafter as P. iterative, adopts the iterative algorithm used in [3]. Thus, the precoder analog part can be obtained by $\mathbf{F}_{R F}=$ column-iterative $\left(\mathbf{H}^{H} \mathbf{W} \mathbf{W}^{H} \mathbf{H}\right)$.

Observe that without hardware constraints, another solution of (9) can be $\mathbf{F}_{R F}=(\overline{\mathbf{U}})_{:, 1: N_{R F_{t}}}$, where $\left[\overline{\mathbf{U}}, \overline{\boldsymbol{\Lambda}}, \overline{\mathbf{U}}^{H}\right]=$ $\operatorname{svd}\left\{\mathbf{H}^{H} \mathbf{W} \mathbf{W}^{H} \mathbf{H}\right\}$. Considering that the approximation of the unconstrained analog precoder by dividing each element by its magnitude is the solution which minimizes the euclidean distance between the unconstrained and phase constrained precoder as proved in [11]. The second solution, named hereafter as P. SVD, can be obtained as follows

$$
\mathbf{F}_{R F}^{*}=\frac{1}{\sqrt{N_{r}}} \boldsymbol{\Psi}\left((\overline{\mathbf{U}})_{:, 1: N_{R F_{r}}}\right)
$$

where the function $\Psi(\mathbf{A})$ returns the entries of the matrix $\mathbf{A} \in \mathbb{C}^{n \times m}$ normalized to magnitude 1, i.e., $(\mathbf{\Psi}(\mathbf{A}))_{i, j}=$ $\frac{\mathbf{A}_{i, j}}{\left\|\mathbf{A}_{i, j}\right\|}, i=1, \ldots, n, j=1, \ldots . m$.

\section{B. Digital part of the hybrid precoder}

The optimization sub-problem related to $\mathbf{F}_{B B}$ can be formulated as

$$
\begin{aligned}
& \mathbf{F}_{B B}^{*}=\underset{\mathbf{F}_{B} B}{\arg \max } \operatorname{tr}\left\{\mathbf{F}_{B B}^{H} \mathbf{M} \mathbf{F}_{B B}\right\} \\
& \text { s.t. }\left\|\left(\mathbf{F}_{R F}\right)_{i, j}\right\|=1 / \sqrt{N_{t}},\|\mathbf{F}\|_{F}^{2} \leq E_{T}
\end{aligned}
$$

where $\mathbf{M}=\mathbf{F}_{R F}^{H} \mathbf{H}^{H} \mathbf{W} \mathbf{W}^{H} \mathbf{H} \mathbf{F}_{R F}$ 
Note that in (11), it is desirable that the product $\mathbf{F}_{B B}^{H} \mathbf{M F}_{B B}$ has its diagonal entries with large values. To reach this desired result, we use the lemma result obtained by [5].

Lemma 1: The optimal structure of the baseband beamformer matrix in (11) is

$$
\mathbf{F}_{B B}=\mathbf{M}^{-1 / 2} \mathbf{U} \mathbf{\Lambda}^{1 / 2}
$$

where $\mathbf{U}$ is a para-unitary matrix such that $\mathbf{U}^{H} \mathbf{U}=\mathbf{I}_{N_{s}}$ and $\boldsymbol{\Lambda}$ is a diagonal matrix. See [5] for more details.

Substituting (12) into the power constraint in (11) yields

$$
\begin{aligned}
\|\mathbf{F}\|_{F}^{2} & \stackrel{\text { def })}{=} \operatorname{Tr}\left\{\mathbf{F}_{R F} \mathbf{F}_{B B} \mathbf{F}_{B B}^{H} \mathbf{F}_{R F}^{H}\right\} \\
& =\operatorname{Tr}\left\{\mathbf{F}_{B B}^{H} \mathbf{F}_{R F}^{H} \mathbf{F}_{R F} \mathbf{F}_{B B}\right\} \\
& =\operatorname{Tr}\left\{\boldsymbol{\Lambda}^{1 / 2} \mathbf{U}^{H} \mathbf{M}^{-1 / 2} \mathbf{F}_{R F}^{H} \mathbf{F}_{R F} \mathbf{M}^{-1 / 2} \mathbf{U} \boldsymbol{\Lambda}^{1 / 2}\right\} \\
& \stackrel{(\text { a) })}{=} \operatorname{Tr}\{\boldsymbol{\Lambda} \tilde{\mathbf{\Lambda}}\} \leq E_{T}
\end{aligned}
$$

where the equality (a) in (13) is reached by considering $\left[\tilde{\mathbf{U}}, \tilde{\mathbf{\Lambda}}, \tilde{\mathbf{U}}^{H}\right]=\operatorname{svd}\left\{\mathbf{M}^{-1 / 2} \mathbf{F}_{R F}^{H} \mathbf{F}_{R F} \mathbf{M}^{-1 / 2}\right\}$ and taking $\mathbf{U}=$ $\tilde{\mathbf{U}}$.

To find the optimum entries values of $\boldsymbol{\Lambda}$ the achievable rate in (6) can be rewritten as

$$
\begin{aligned}
& R=\log _{2}\left|\mathbf{I}_{N_{s}}+\mathbf{K}^{-1} \mathbf{W}^{H} \mathbf{H F F} \mathbf{F}^{H} \mathbf{H}^{H} \mathbf{W}\right| \\
& \stackrel{(a)}{=} \log _{2} \mid \mathbf{I}_{N_{s}}+\mathbf{U}^{H} \mathbf{M}^{-1 / 2} \mathbf{F}_{R F}^{H} \mathbf{H}^{H} \mathbf{W K}^{-1} \ldots \\
& \ldots \mathbf{W}^{H} \mathbf{H F}_{R F} \mathbf{M}^{-1 / 2} \mathbf{U} \boldsymbol{\Lambda} \\
& \stackrel{(\mathrm{b})}{\approx} \log _{2}\left|\mathbf{I}_{N_{s}}+\mathbf{D} \boldsymbol{\Lambda}\right|=\sum_{i=1}^{N_{s}} \log _{2}\left(1+d_{i} \lambda_{i}\right)
\end{aligned}
$$

The equality (a) in (14) is easily reached by making use of (12) and the property $\left|\mathbf{I}_{A B}+\mathbf{A B}\right|=\left|\mathbf{I}_{B A}+\mathbf{B A}\right|$, whereas the approximation (b) is obtained by considering $\mathbf{D}=$ $\operatorname{diag}\left\{\mathbf{U}^{H} \mathbf{M}^{-1 / 2} \mathbf{F}_{R F}^{H} \mathbf{H}^{H} \mathbf{W} \mathbf{K}^{-1} \mathbf{W}^{H} \mathbf{H} \mathbf{F}_{R F} \mathbf{M}^{-1 / 2} \mathbf{U}\right\}$. Furthermore, $d_{i}$ and $\lambda_{i}$ represent the $i$-th diagonal entry of the matrices $\mathbf{D}$ and $\boldsymbol{\Lambda}$, respectively.

Note that the assumption for the approximation (b) is more appropriated when the product $\mathbf{W} \mathbf{W}^{H} \approx I_{N_{r}}$. This result can be obtained by taking the digital part of the hybrid combiner as para-unitary matrix (as it will be proposed in this letter). The product $\mathbf{W}_{R F} \mathbf{W}_{R F}^{H}$ does not affect this assumption because it tends to an identity matrix as the number of antennas increases as proved in [12].

From (14) and (13) the following optimization problem is considered

$$
\begin{aligned}
& \boldsymbol{\Lambda}^{*}=\underset{\boldsymbol{\Lambda}}{\arg \min }-\sum_{i=1}^{N_{s}} \log _{2}\left(1+d_{i} \lambda_{i}\right) \\
& \text { s.t. } \sum_{i=1}^{N_{s}} \lambda_{i} \tilde{\lambda}_{i} \leq E_{T}, \lambda_{i} \geq 0
\end{aligned}
$$

where $\tilde{\lambda}_{i}$ is the $i$-th diagonal entry of $\tilde{\Lambda}$. A very similar optimization problem was derived in [5], however the authors considered $d_{i}$ as a constant, whose value is not explained nether optimum. The deduction of (14) clearly evidences $d_{i}$ is variable and its value is delimited by $\mathbf{D}$. Therefore, a more proper solution of (15) is given by the following lemma.

Lemma 2: The optimization problem in (15) is convex and its optimal solutions is given by

$$
\lambda_{i}=\left[\frac{1}{\tilde{\lambda}_{i} \beta \ln 2}-\frac{1}{d_{i}}\right]^{\dagger}
$$

where $\beta$ is the Lagrangian multiplier, and $a^{\dagger}=\max \{a, 0\}$ returns the maximum value between a and zero.

Proof: In (15), the constraint is linear with respect to $\tilde{\lambda}_{i}$, which is convex, and the objective function is concave as proved in [5]. Equation (15) can be easily solved by defining a Lagrangian function, whose solution is (16) and $\beta=\frac{d_{\max } N_{s}}{\ln 2\left(d_{\max } E_{T}+N_{s} \tilde{\lambda}_{N_{s}}\right)}$, where $d_{\max }$ is the maximum entry taken from $\mathbf{D}$. Finally, to ensure the transmission power constraint is satisfied, a normalization constant is computed such that $\|\mathbf{F}\|_{F}^{2}=E_{T}$

\section{HYBRID COMBINER DESIGN}

The optimization problem described by (9) increases the system rate, given $\mathbf{H}$ and $\mathbf{W}$, through $\mathbf{F}_{R F}$. This solution can be further optimized by selecting a proper choice of $\mathbf{W}$ as

$$
\begin{gathered}
\mathbf{W}^{*}=\underset{\mathbf{W}}{\arg \max } \operatorname{tr}\left\{\mathbf{F}_{R F}^{H} \mathbf{H}^{H} \mathbf{W} \mathbf{W}^{H} \mathbf{H F}_{R F}\right\} \\
\text { s.t. }\left\|\left(\mathbf{W}_{R F}\right)_{i, j}\right\|=1 / \sqrt{N_{r}}
\end{gathered}
$$

Below follows some manipulations of the target function in the above problem

$$
\begin{aligned}
\operatorname{tr}\left\{\mathbf{F}_{R F}^{H} \mathbf{H}^{H} \mathbf{W} \mathbf{W}^{H} \mathbf{H} \mathbf{F}_{R F}\right\} & =\operatorname{tr}\left\{\mathbf{W}^{H} \mathbf{H} \mathbf{F}_{R F} \mathbf{F}_{R F}^{H} \mathbf{H}^{H} \mathbf{W}\right\} \\
& \stackrel{(\mathrm{a})}{\approx} \operatorname{tr}\left\{\mathbf{W}^{H} \mathbf{H} \mathbf{H}^{H} \mathbf{W}\right\}
\end{aligned}
$$

The approximation ( $a$ ) considers that $\mathbf{F}_{R F} \mathbf{F}_{R F}^{H} \approx \mathbf{I}_{N_{t}}$. As stated before, reference [12] has shown that this product tends to an identity matrix as the number of antennas increases. Therefore, the analog combiner design problem can be formulated as follows

$$
\begin{gathered}
\mathbf{W}^{*}=\underset{\mathbf{W}}{\arg \max } \operatorname{tr}\left\{\mathbf{W}^{H} \mathbf{H} \mathbf{H}^{H} \mathbf{W}\right\} \\
\text { s.t. }\left\|\left(\mathbf{W}_{R F}\right)_{i, j}\right\|=1 / \sqrt{N_{r}}
\end{gathered}
$$

To optimize the diagonal entries of the product $\mathbf{W}^{H} \mathbf{H} \mathbf{H}^{H} \mathbf{W}$, we adopt the methodology proposed in [13]. The digital part of the hybrid combiner is computed as $\mathbf{W}_{B B}=\mathbf{U}_{c}$, where $\left[\mathbf{U}_{c}, \boldsymbol{\Lambda}_{c}, \mathbf{U}_{c}^{H}\right]=$ $\operatorname{svd}\left\{\mathbf{W}_{R F}^{H} \mathbf{H} \mathbf{H}^{H} \mathbf{W}_{R F}\right\}$. Then, substituting this solution in (19), the following problem can be formulated to find the proper solution of the analog beamformer of the hybrid combiner 


$$
\begin{gathered}
\mathbf{W}_{R F}^{*}=\underset{\mathbf{W}_{R F}}{\arg \max } \operatorname{tr}\left\{\mathbf{W}_{R F}^{H} \mathbf{H} \mathbf{H}^{H} \mathbf{W}_{R F}\right\} \\
\text { s.t. }\left\|\left(\mathbf{W}_{R F}\right)_{i, j}\right\|=1 / \sqrt{N_{r}}
\end{gathered}
$$

Observe that (20) can be solved by making use of the methodology applied in the analog part of P. SVD (see (10)). Therefore, the analog part of the hybrid combiner can be computed as

$$
\mathbf{W}_{R F}^{*}=\frac{1}{\sqrt{N_{r}}} \mathbf{\Psi}\left(\left(\tilde{\mathbf{U}}_{c}\right)_{:, 1: N_{R F_{r}}}\right)
$$

where $\left[\tilde{\mathbf{U}}_{c}, \tilde{\boldsymbol{\Lambda}}_{c}, \tilde{\mathbf{U}}_{c}^{H}\right]=\operatorname{svd}\left\{\mathbf{H H}^{H}\right\}$.

\section{Numerical Results}

The mmWave channels are generated with $N_{p}=8$ multipaths components, the azimuth and elevation departure angles values are given by a random variable with uniform distribution in the interval of $(0,2 \pi)$ and $(0, \pi)$, respectively. The UPAs have square formats for both transmitter and receiver, i.e., $N_{t_{h}}=N_{t_{v}}=\sqrt{N}_{t}$ and $N_{r_{h}}=N_{r_{v}}=\sqrt{N}_{r}$. The minimum allowed setting for the number of RF chains is used for both the BS and MS, so that $N_{R F_{t}}=N_{R F_{r}}=N s$. The total energy available at the BS, $E_{T}$, is equal to $N_{s}$. The results are averaged over $10^{3}$ channel realizations. The following scenarios are considered: $\left(\mathrm{S}_{1}\right) N_{t}=144, N_{r}=16$, and $N_{s}=4 ;\left(\mathrm{S}_{2}\right) N_{t}=255, N_{r}=16$, and $N_{s}=4 ;$ and $\left(\mathrm{S}_{3}\right)$ $\mathrm{SNR}=10 \mathrm{~dB}, N_{r}=16, N_{s}=4$, and $N_{t}$ as variable. Fig. 1 presents the achievable rate results obtained by considering $\mathrm{S}_{1}$ and $\mathrm{S}_{2}$, whereas Fig. 2 shows the numerical results related to $\mathrm{S}_{3}$.

For comparison purposes, an optimal fully-digital technique is used as an evaluation benchmark. This digital technique computes a fully-digital matched filter (MF) as a precoder and a para-unitary matrix as a combiner, i.e., $\mathbf{F}=n c \mathbf{H}^{H}$, where $n c$ is computed such that $\|\mathbf{F}\|^{2}=E_{T}$, and $\mathbf{W}=\tilde{\mathbf{U}}_{c}$. Since the MF can maximize the SNR, it reaches an optimum spectrum efficiency that represents an optimum benchmark for single-user MIMO scenarios [6].

In Fig. 1, it is noted that P. iterative and P. SVD achieve very similar performance in both scenarios, $S_{1}$ and $S_{2}$. However, P. iterative has a slight gain relative to P. SVD, which is produced through optimizing the analog beamformer of the transmitter proposed according to [10]. Note that any of the proposed solutions not only surpasses the considered hybrid techniques but also the considered fully digital technique.

Fig. 2 clearly evidences the advantages of the proposed methodology. Note that there is a performance gap between the considered hybrid and digital techniques. However, the proposed methodology eliminates this gap and obtains a slight gain in relation to Fully-digital. It is necessary to highlight that the performance obtained by the considered fully-digital technique does not represent the maximum achievable performance. However, the use of MF is an excellent approach to optimize the SNR and increase the spectral efficiency in SU-MIMO scenarios. Therefore, the obtained performance is difficult to be overcome by hybrid techniques as evidenced

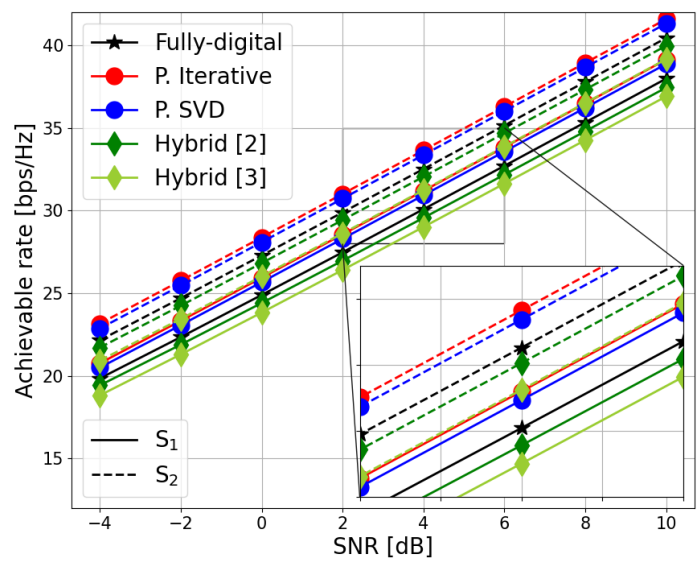

Fig. 1. Achievable rate comparison considering $\mathrm{S}_{1}$ and $\mathrm{S}_{2}$

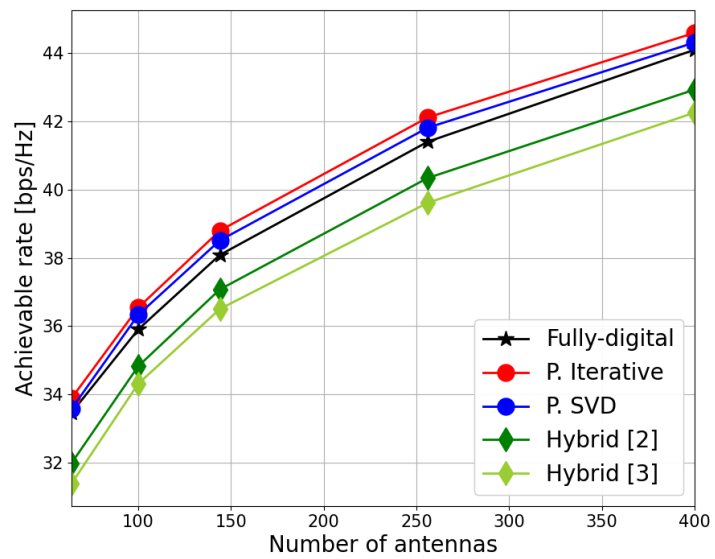

Fig. 2. Achievable rate comparison considering $\mathrm{S}_{3}$

in [7]-[9]. Note that the proposed methodology performs multiple optimizations to reach a slight gain over Fullydigital. The proposed RF and passband beamformer of both transmitter and receiver are coordinated with each other to further optimize the spectral efficiency of the system.

\section{CONCLUSIONS}

We proposed a method to increase the spectral efficiency in the mmWave massive SU-MIMO system. The proposed method formulates four different optimization subproblems by making use of a hierarchical strategy. For these subproblems, two solutions were put forward, P. Iterative and P. SVD. Numerical results evidenced the proposed optimization by parts is well addressed due to the largest rate values were got by our proposals. The main feature of the proposed methodology relies on the coordination of the proposed RF and passband beamformer of both the transmitter and the receiver. This fact produced that the two proposed hybrid processing even surpass the considered fully-digital technique. 


\section{REFERENCES}

[1] R. W. Heath, N. Gonzalez-Prelcic, S. Rangan, W. Roh, and A. M. Sayeed, "An overview of signal processing techniques for millimeter wave MIMO systems," IEEE journal of selected topics in signal processing, vol. 10, no. 3, pp. 436-453, 2016.

[2] A. Li and C. Masouros, "Hybrid precoding and combining design for millimeter-wave multi-user MIMO based on SVD," in Communications (ICC), 2017 IEEE International Conference on. IEEE, 2017, pp. 1-6.

[3] Á. J. Ortega, R. Sampaio-Neto, and R. P. David, "Hybrid precoded index modulation in downlink mmwave MU-MIMO systems," in 2019 International Conference on Computing, Networking and Communications (ICNC). IEEE, 2019, pp. 329-333.

[4] D. H. Nguyen, L. B. Le, T. Le-Ngoc, and R. W. Heath, "Hybrid MMSE precoding and combining designs for mmwave multiuser systems," IEEE Access, vol. 5, pp. $19167-19181,2017$.

[5] Q. Hou, R. Wang, E. Liu, and D. Yan, "Hybrid precoding design for MIMO system with one-bit ADC receivers," IEEE Access, vol. 6, pp. $48478-48488,2018$.

[6] E. Kabalci and Y. Kabalci, From smart grid to internet of energy. Academic Press, 2019.

[7] W. Wang, H. Yin, X. Chen, and W. Wang, "Performance loss of hybrid beamforming with imperfect phase shifters in millimeter wave systems," in 2018 IEEE 88th Vehicular Technology Conference (VTC-Fall). IEEE, 2018, pp. $1-5$.

[8] N. Li, Z. Wei, H. Yang, X. Zhang, and D. Yang, "Hybrid precoding for mmwave massive mimo systems with partially connected structure," IEEE Access, vol. 5, pp. $15142-15$ 151, 2017.

[9] F. Sohrabi and W. Yu, "Hybrid analog and digital beamforming for mmwave ofdm large-scale antenna arrays," IEEE Journal on Selected Areas in Communications, vol. 35, no. 7, pp. 1432-1443, 2017.

[10] C. Hu, J. Liu, X. Liao, Y. Liu, and J. Wang, "A novel equivalent baseband channel of hybrid beamforming in massive multiuser MIMO systems," IEEE Commun. Lett., vol. PP, no. 99, pp. 1-1, 2017.

[11] S. Park, J. Park, A. Yazdan, and R. W. Heath, "Exploiting spatial channel covariance for hybrid precoding in massive MIMO systems," IEEE Transactions on Signal Processing, vol. 65, no. 14, pp. 38183832, 2017.

[12] F. Sohrabi and W. Yu, "Hybrid digital and analog beamforming design for large-scale antenna arrays," IEEE Journal of Selected Topics in Signal Processing, vol. 10, no. 3, pp. 501-513, 2016.

[13] A. J. Ortega, R. Sampaio-Neto, and R. D. Pereira, "On hybrid precoder/combiner for downlink mmWave massive MU-MIMO systems," Preprint Researchgate DOI: 10.13140/RG.2.2.36339.58405, 2019. 